\title{
Experimental Research of Flow-Coupled Secondary Regulation System Using Nonlinear Controller
}

\author{
Yang Xin and Deng Kai \\ Department of Physical Science and Electronic Engineering, Yibin University, \\ Yibin 644000, China \\ Yang_xin_2002@163.com
}

\begin{abstract}
A nonlinear mathematical model of the flow-coupled secondary regulation system was established. Then, the exact feedback linearization was applied to transform the system into a linear one by coordinates transformation and state-space feedback based on its nonlinear model. Furthermore, the zero dynamics was analyzed to prove the stability of the system so as to verify the feasibility of the exact linearization control scheme. And the $L Q R$ controller was design on the basis of the exact linearization control scheme. Finally, the experimental results show that the Flow-coupled secondary regulation system using the LQR controller based on the exact linearization control scheme has good performance and does better than PID controller when it works far from zero point.
\end{abstract}

Keywords: Flow-coupled system, nonlinear model, zero dynamics analysis, nonlinear control, experimental research

\section{Introduction}

The Constant Pressure System (CPS) was paid more attention because it could avoid throttle loss and overflow loss to improve system efficiency and could connect some different loads. However, the CPS was not suitable to connect the fixed displacement actuators, such as reciprocating cylinder. The flow-coupled secondary regulation system is a new type of energy-saving system that is different from the CPS working as constant pressure rail [1-2]. Its system pressure was variable according to the load. Hence, it can connect the fixed displacement actuators. With the energy sources being critical, the system becomes more and more important both in theory and practice. In general, the system was linearized at a certain point using Taylor series expansion. The performance and effectiveness of a controller based on a linear model was generally guaranteed only during small deviations from the nominal operating point. And the traditional control laws based on linearized models were limited. Recently, the exact linearization method, in witch the system can be exactly transformed into a linear system by adding an appropriate control input and using new coordinates, has been developed and applied to nonlinear control systems [3-4]. For example, Na Woon and Gao Zhaohui took advantage of exact linearization control to PEM fuel cells and BUCK converter respectively [5-6]. In this paper, the nonlinear model of the flow-coupled secondary regulation system was developed. Then, the exact feedback linearization was applied to transform the system into a linear one by coordinates transformation and state-space feedback based on its nonlinear model. Furthermore, the zero dynamics was analyzed to verify the validity of the controller. Finally, the experimental results show that the system using the LQR controller based on the exact linearization control scheme has good control performance and is irrelevant to work point. 


\section{System Model}

The flow-coupled secondary regulation system was shown in Figure.1. The time domain representation was given by the following dynamic equations:

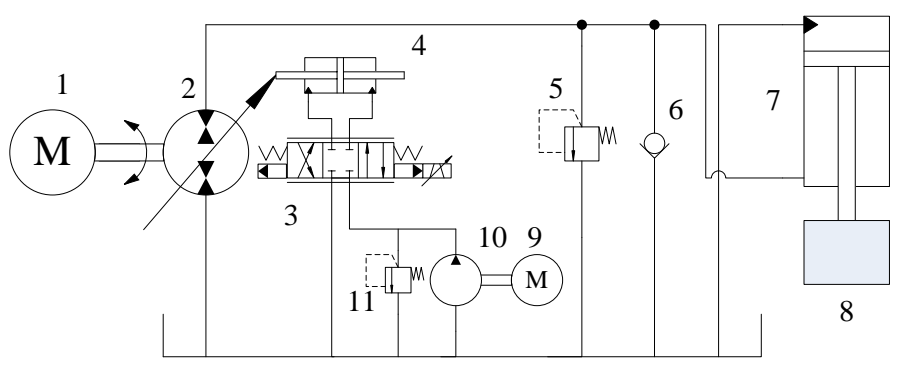

1-Motor; 2-Hydraulic Pump/Motor; 3-Electro-Hydraulic Servo Valve; 4-Variable Cylinder; 5-Safety Valve; 6-Check Valve; 7-Load Cylinder; 8-Load; 9-Control Motor; 10-Control

Pump; 11-Relief Valve

\section{Figure 1. Schematic Diagram of Flow-Coupled Secondary Regulation System}

Servo amplifier equation can be expressed as follows:

$$
i=K_{i} u
$$

Equation between displacement of servo valve and input current can be given as:

$$
x_{v}=K_{s} i
$$

Inlet flow rate of variable cylinder can be written as:

$$
q_{1}=C_{d} w x_{v} \sqrt{\frac{1}{\rho}\left(p_{c}-p_{1}\right)}
$$

Outlet flow rate of variable cylinder can be written as:

$$
q_{2}=C_{d} w x_{v} \sqrt{\frac{2}{\rho} p_{2}}
$$

Continuity equation of inlet chamber of variable cylinder can be written as:

$$
q_{1}-C_{i c}\left(p_{1}-p_{2}\right)-C_{e c} p_{1}=A_{g} \frac{d y}{d t}+\frac{V_{1}}{\beta_{e}} \frac{d p_{1}}{d t}
$$

Where $V_{1}=V_{0}+A_{g} y$

Continuity equation of outlet chamber of variable cylinder can be written as:

$$
C_{i c}\left(p_{1}-p_{2}\right)-C_{e c} p_{2}-q_{2}=-A_{g} \frac{d y}{d t}+\frac{V_{2}}{\beta_{e}} \frac{d p_{2}}{d t}
$$

Where $V_{2}=V_{0}-A_{g} y$

Force balance equation of variable cylinder can be written as:

$$
A_{g}\left(p_{1}-p_{2}\right)=m \frac{d^{2} y}{d t^{2}}+B_{c} \frac{d y}{d t}+K y
$$

Displacement equation of hydraulic pump/motor can be written as:

$$
D=\frac{y}{y_{\text {max }}} D_{\text {max }}
$$

Continuity equation of hydraulic pump/motor can be written as: 


$$
\omega D-C_{t} p_{L}=A v+\frac{V}{4 \beta_{e}} \frac{d p_{L}}{d t}
$$

Force balance equation of load cylinder can be written as:

$$
p_{L} A+m_{p} g=\left(M+m_{p}\right) \frac{d v}{d t}+B v+M g
$$

Where symbols denote as follows:

$u$ : input voltage $(\mathrm{V})$;

$i$ : input current $(\mathrm{A})$;

$K_{\mathrm{i}}$ : amplifier gain $(\mathrm{A} / \mathrm{V})$;

$K_{\mathrm{s}}$ : coefficient of servo valve $(\mathrm{m} / \mathrm{A})$;

$q_{1}$ : flow rate of inlet chamber of the variable cylinder $\left(\mathrm{m}^{3} / \mathrm{s}\right)$;

$q_{2}$ : flow rate of outlet chamber of the variable cylinder $\left(\mathrm{m}^{3} / \mathrm{s}\right)$;

$C_{\mathrm{d}}$ : discharge coefficient;

$y$ : displacement of cylinder $(\mathrm{m})$;

$w$ : area grades of servo valve spool (m);

$x_{\mathrm{v}}$ : displacement of servo valve spool (m);

$\rho$ : density of oil $(\mathrm{kg} / \mathrm{m} 3)$;

$p_{\mathrm{c}}$ : inlet chamber pressure of variable cylinder $(\mathrm{Pa})$;

$p_{1}$ : outlet chamber pressure of variable cylinder $(\mathrm{Pa})$;

$p_{2}$ : supply pressure to valve $(\mathrm{Pa})$;

$p_{\mathrm{L}}$ : system pressure $(\mathrm{Pa})$;

$A_{g}:$ area of variable cylinder piston $\left(\mathrm{m}^{2}\right)$;

$C_{\mathrm{ic}}$ : internal leakage coefficient of variable cylinder $(\mathrm{m} \cdot \mathrm{N} / \mathrm{s})$;

$C_{\mathrm{e}}$ : external leakage coefficient of variable cylinder $(\mathrm{m} \cdot \mathrm{N} / \mathrm{s})$;

$V_{1}$ : volume of forward chamber $\left(\mathrm{m}^{3}\right)$;

$V_{2}$ : volume of return chamber $\left(\mathrm{m}^{3}\right)$;

$V_{0}$ :original Volume of variable cylinder $\left(\mathrm{m}^{3}\right)$;

$A$ : effective area of load cylinder (m2);

$C_{\mathrm{t}}$ : total leakage coefficient of load cylinder $\left(\left(\mathrm{m}^{3} / \mathrm{s}\right) / \mathrm{Pa}\right)$;

$\beta_{\mathrm{e}}$ : volume elasticity modulus of oil $\left(\mathrm{N} / \mathrm{m}^{2}\right)$;

$m$ : total mass of piston and swash plate reffered to piston $(\mathrm{kg})$;

$B_{\mathrm{c}}$ : damp coefficient of cylinder $(\mathrm{N} /(\mathrm{m} \cdot \mathrm{s}))$;

$K$ : load spring gradient $(\mathrm{N} / \mathrm{m})$;

$D$ : displacement of secondary unit $\left(\mathrm{m}^{3} / \mathrm{rad}\right)$;

$D_{\max }$ : the maximum displacement of secondary unit $\left(\mathrm{m}^{3} / \mathrm{rad}\right)$;

$y_{\max }$ : the maximum displacement of cylinder $(\mathrm{m})$;

$\omega$ :angular velocity of hydraulic pump/motor $(\mathrm{rad} / \mathrm{s})$;

$V$ : volume of load cylinder $\left(\mathrm{m}^{3}\right)$;

$v$ :piston velocity $(\mathrm{m} / \mathrm{s})$;

$B$ : viscous damping coefficient $(\mathrm{N} /(\mathrm{m} \cdot \mathrm{s}))$;

$M_{\mathrm{p}}$ : mass of load cylinder piston $(\mathrm{kg})$;

$M$ : load mass (kg)

Let $x_{1}=v, x_{2}=p_{L}, x_{3}=y, x_{4}=\dot{y}, x_{5}=p_{1}, x_{6}=p_{2}$, the state-space equation of the system is:

Where

$$
\left\{\begin{array}{l}
\dot{x}=f(x)+g(x) u \\
y=h(x)
\end{array}\right.
$$




$$
\begin{aligned}
& f(x)=\left(\begin{array}{l}
-\frac{B}{M+m_{p}} x_{1}+\frac{A}{M+m_{p}} x_{2}+\frac{m_{p}-M}{M+m_{p}} g \\
-\frac{4 \beta_{e} A}{V} x_{1}-\frac{4 \beta_{e} C_{t}}{V} x_{2}+\frac{4 \beta_{e} \omega D_{\max }}{V x_{3 \max }} x_{3} \\
x_{4} \\
-\frac{K}{m} x_{3}-\frac{B_{c}}{m} x_{4}+\frac{A_{g}}{m} x_{5}-\frac{A_{g}}{m} x_{6} \\
-\frac{\beta_{e} A_{g}}{V_{0}+A_{g} x_{3}} x_{4}-\frac{\beta_{e}\left(C_{i c}+C_{e c}\right)}{V_{0}+A_{g} x_{3}} x_{5}+\frac{\beta_{e} C_{i c}}{V_{0}+A_{g} x_{3}} x_{6} \\
\frac{\beta_{e} A_{g}}{V_{0}-A_{g} x_{3}} x_{4}+\frac{\beta_{e} C_{i c}}{V_{0}-A_{g} x_{3}} x_{5}-\frac{\beta_{e}\left(C_{i c}+C_{e c}\right)}{V_{0}-A_{g} x_{3}} x_{6} \\
0 \\
0 \\
0 \\
0 \\
0(x)=\left(\begin{array}{l}
\frac{\beta_{e} C_{d} w K_{i} K_{s}}{V_{0}+A_{g} x_{3}} \sqrt{\frac{2}{\rho}\left(p_{c}-x_{5}\right)} \\
-\frac{\beta_{e} C_{d} w K_{i} K_{s}}{V_{0}-A_{g} x_{3}} \sqrt{\frac{2}{\rho} x_{6}}
\end{array}\right) \\
x_{1}
\end{array}\right)
\end{aligned}
$$

\section{Exact Linearization}

This mathematical model (11) is a standard model of affine nonlinear systems. Here $x \in R^{n} f, g, h$ are smooth. This system is said to have relative degree $r$ at a point $x_{0}$, if $r$ is the smallest integer such that

$$
\begin{aligned}
& L_{g} L_{f}^{i} h(x) \equiv 0, \forall x \in U, i=0, \cdots, r-2 ; \\
& L_{g} L_{f}^{r-1} h(x) \neq 0
\end{aligned}
$$

And all $x$ in a neighborhood of $x_{0}$. Where $L_{f}$ and $L_{g}$ are the Lie derivatives with respect to $f$ and $g$. The Lie derivative is a convenient notation, examples being

$$
\begin{aligned}
& L_{f} h(x)=\sum_{i=1}^{n} \frac{\partial h}{\partial x_{i}} f_{i}(x) \\
& L_{g} h(x)=\sum_{i=1}^{n} \frac{\partial h}{\partial x_{i}} g_{i}(x)
\end{aligned}
$$

We can obtain: 


$$
\begin{aligned}
& L_{g} h(x)=0 \\
& L_{g} L_{f} h(x)=0 \\
& L_{g} L_{f}^{2} h(x)=0 \\
& L_{g} L_{f}^{3} h(x)=0 \\
& L_{g} L_{f}^{4} h(x) \neq 0
\end{aligned}
$$

According to the definition of $r$, we can get $r=5<6$ (dimension of the system). And we can select a function $\xi(x)$ which has to satisfy with

$$
L_{g} \xi(x)=\langle d \xi(x), g\rangle=0 \text { and }
$$

One of roots is as follows:

$$
\operatorname{rank}\left(\begin{array}{l}
h(x) \\
L_{f} h(x) \\
L_{f}^{2} h(x) \\
L_{f}^{3} h(x) \\
L_{f}^{4} h(x) \\
\xi(x)
\end{array}\right)=6
$$

$$
\xi_{1}(x)=\left(V_{0}-A_{g} x_{3}\right) \sqrt{x_{6}}-\left(V_{0}+A_{g} x_{3}\right) \sqrt{\left(p_{c}-x_{5}\right)}
$$

And we can get the nonlinear coordinate transformation:

$$
\phi: x \rightarrow\left(\begin{array}{l}
h(x) \\
L_{f} h(x) \\
L_{f}^{2} h(x) \\
L_{f}^{3} h(x) \\
L_{f}^{4} h(x) \\
\xi(x)
\end{array}\right)
$$

This transformation is local differential homeomorphism since the Jacobi matrix of $\phi(x)$ is not singular. let

$$
\begin{gathered}
z=\left(\begin{array}{l}
h(x) \\
L_{f} h(x) \\
L_{f}^{2} h(x) \\
L_{f}^{3} h(x) \\
L_{f}^{4} h(x)
\end{array}\right) \\
\xi=z_{\gamma+1}
\end{gathered}
$$

We can get the exact linearization controller is:

$$
u=\frac{1}{L_{g} L_{f}^{4} h(x)}\left(-L_{f}^{5} h(x)+v\right)=\alpha(x)+\beta(x) v
$$




\section{Zero DynamicsAnalysis}

The zero outputs have to analysis for $r<n$. when there exists $y(t)=0$, system states are limited within a area as follows:

$$
\dot{z}_{1}(t)=\dot{z}_{2}(t)=\cdots=\dot{z}_{\gamma}(t)=0
$$

Input $u(t)$ is one of the roots of follow equation.

$$
0=b(0, \xi)+a(0, \xi) u(t)
$$

Hence, $\xi(t)$ is controlled by:

$$
\dot{\xi}(t)=q(0, \xi(t))
$$

So we can obtain:

$$
u(t)=\frac{b(0, \xi(t))}{a(0, \xi(t))}
$$

Therefore, the dynamics of eq. (16) is the zero dynamics of the system. If the zero dynamics is stable, the nonlinear system is locally stable. The inner states, $\xi$, are not observable due to state feedback. However, not only the external states have good performance, but also the inner states have to be stable with regard to an exact linearization system. That is let $\dot{\xi}=L_{f} \xi(x)$ is stable. We can get the linearized matrix according to eq. (17):

$$
\frac{\partial q}{\partial \xi}(0,0)
$$

If the eigenvalue of eq. (19) are within $C_{-}^{0}$, the system is locally stable. So we can obtain the zero dynamics equation:

$$
\begin{aligned}
q=\dot{\xi}_{1}= & -A_{g}\left(\sqrt{\left(p_{c}-x_{5}\right)}+\sqrt{x_{6}}\right) x_{4}+\frac{-\beta_{e} A_{g} x_{4}-\beta_{e}\left(C_{i c}+C_{e c}\right) x_{5}+\beta_{e} C_{i c} x_{6}}{2 \sqrt{\left(p_{c}-x_{5}\right)}}+ \\
& \frac{\beta_{e} A_{g} x_{4}+\beta_{e} C_{i c} x_{5}-\beta_{e}\left(C_{i c}+C_{e c}\right) x_{6}}{2 \sqrt{x_{6}}}
\end{aligned}
$$

Table 1. Specifications of Flow-Coupled Secondary Regulation System

\begin{tabular}{cccccc}
\hline Specifications & unit & value & Specifications & Unit & value \\
\hline$A_{\mathrm{g}}$ & $\mathrm{m}^{2}$ & $1.78 \times 10^{-3}$ & $\beta_{\mathrm{e}}$ & $\mathrm{Pa}$ & $6.9 \times 10^{8}$ \\
$K_{\mathrm{s}}$ & $\mathrm{m} / \mathrm{A}$ & $1.5 \times 10^{-5}$ & $C_{\mathrm{ic}}$ & $\mathrm{m} \cdot \mathrm{N} / \mathrm{s}$ & $2.4 \times 10^{-11}$ \\
$C_{\mathrm{d}}$ & - & 0.61 & $C_{\mathrm{ec}}$ & $\mathrm{m} \cdot \mathrm{N} / \mathrm{s}$ & $7.3 \times 10^{-13}$ \\
$w$ & $\mathrm{~m}$ & $1.16 \times 10^{-2}$ & $V_{0}$ & $\mathrm{~m}^{3}$ & $5 \times 10^{-5}$ \\
$\rho$ & $\mathrm{kg} / \mathrm{m}^{3}$ & 900 & $m$ & $\mathrm{Kg}$ & 1 \\
$y_{\max }$ & $\mathrm{m}$ & 0.015 & $B_{c}$ & $\mathrm{~N} \cdot \mathrm{s} / \mathrm{m}$ & 500 \\
$C_{\mathrm{t}}$ & $\mathrm{m} \cdot \mathrm{N} / \mathrm{s}$ & $3 \times 10^{-11}$ & $K$ & $\mathrm{~N} / \mathrm{m}$ & $1.66 \times 10^{5}$ \\
$A$ & $\mathrm{~m}^{2}$ & $2.82 \times 10^{-3}$ & $M$ & $\mathrm{Kg}$ & 2000 \\
$V$ & $\mathrm{~m}^{3}$ & $10^{-3}$ & $B$ & $\mathrm{~N} \cdot \mathrm{s} / \mathrm{m}$ & 1000 \\
$\omega$ & $\mathrm{Rad} / \mathrm{s}$ & 314 & $D_{\max }$ & $\mathrm{m} / \mathrm{rad}$ & $6.37 \times 10^{-6}$ \\
\hline
\end{tabular}

The specifications were shown in Table 1 . Then zero dynamics was got.

$$
\frac{\partial q}{\partial \xi}(0,0)=-0.1
$$

Hence, the exact linearization controller was valid and can be expressed as: 


$$
\begin{aligned}
& u=\frac{2.04 \times 10^{12} x_{3} x_{4}+5 \times 10^{4} x_{2} x_{4}-1.25 \times 10^{12} x_{1} x_{4}^{2}+82.3 x_{2} x_{5}+1.52 \times 10^{-9} x_{1}+6.59 \times 10^{9} x_{3} x_{5}-4.08 \times 10^{9} x_{1} x_{4} x_{5}}{-9.7 \times 10^{-7}\left(\frac{-3.45 \sqrt{x_{6}}}{5 \times 10^{-5}-1.785 \times 10^{-3} x_{4}}-\frac{3.45 \sqrt{2 \times 10^{6}-x_{7}}}{5 \times 10^{-5}+1.785 \times 10^{-3} x_{4}}\right)}+ \\
& \frac{-1.61 \times 10^{13} x_{3} x_{4}^{3}-5.71 \times 10^{4} x_{2} x_{4}^{3}+1.52 \times 10^{13} x_{1} x_{4}^{4}+6.21 \times 10^{3} x_{2} x_{4}^{2}+6.21 \times 10^{3} x_{2} x_{4} x_{5}-1.29 \times 10^{12} x_{1} x_{4}+3.9 \times 10^{6} x_{1} x_{5}^{2}}{-9.7 \times 10^{-7}\left(\frac{-3.45 \sqrt{x_{6}}}{5 \times 10^{-5}-1.785 \times 10^{-3} x_{4}}-\frac{3.45 \sqrt{2 \times 10^{6}-x_{7}}}{5 \times 10^{-5}+1.785 \times 10^{-3} x_{4}}\right)}- \\
& -596\left(x_{6}-x_{7}\right)-9.7 \times 10^{-7}\left(\frac{1.23 \times 10^{6} x_{5}+1.66 \times 10^{-2}\left(x_{6}-x_{7}\right)}{5 \times 10^{-5}-1.785 \times 10^{-3} x_{4}}+\frac{1.23 \times 10^{6} x_{5}+1.66 \times 10^{-2}\left(x_{6}-x_{7}\right)}{5 \times 10^{-5}+1.785 \times 10^{-3} x_{3}}\right)+v \\
& -9.7 \times 10^{-7}\left(\frac{-3.45 \sqrt{x_{6}}}{5 \times 10^{-5}-1.785 \times 10^{-3} x_{4}}-\frac{3.45 \sqrt{2 \times 10^{6}-x_{7}}}{5 \times 10^{-5}+1.785 \times 10^{-3} x_{4}}\right)
\end{aligned}
$$

Now, the system became linear after exact linearization (shown in eq. 21 ).

$$
y^{(5)}=v
$$

We can synthesize the new system using linear control theory.

\section{Design of LQR Controller Using Dominant-Pole Algorithm}

According to the optimal control law based on linear quadratic regulator [7], the performance index is given:

$$
J=\frac{1}{2} \int_{0}^{\infty}\left(X^{T} Q X+v^{T}(t) R v\right) d t
$$

Where, $Q$ and $R$ are weight matrix. They are limitary symmetrical positive definite matrix during the zone of $[0, \infty]$. Meanwhile, they can be obtained using dominant-pole algorithm. Therefore, the goal is to seek for the optimal control input $v(t)$ to minimize performance index $J$. The index $J$ is the smallest when $v^{*}=-K^{*} Z$ according to the optimal control law of linear quadratic regulator.

Where feedback gains $K^{*}$ can be determined as

$$
K^{*}=\left[K_{1}{ }^{*}, K_{2}{ }^{*}, K_{3}{ }^{*}, K_{4}{ }^{*}, K_{5}{ }^{*}\right]=R^{-1} B^{T} P
$$

$P$ is the root of Riccati algebra equation:

$$
P A+A^{T} P-P B R^{-1} B^{T} P+Q=0
$$

The resulting controller is derived:

$$
u=\frac{1}{L_{g} L_{f}^{n-1} h(x)}\left[-L_{f}^{n} h(x)-K^{*} Z\right]
$$

According to engineering practice, the dominant poles were placed:

$$
s_{1,2}=-3 \pm 1.8 j
$$

Hence, the weight matrix and feedback gains were got:

$$
\begin{aligned}
& Q=\left[\begin{array}{lllll}
81 & 43.2 & 9 & 0 & 0 \\
43.2 & 23.04 & 4.8 & 0 & 0 \\
90 & 4.8 & 1 & 0 & 0 \\
0 & 0 & 0 & 0 & 0 \\
0 & 0 & 0 & 0 & 0
\end{array}\right], R=\mathrm{I} \\
& K^{*}=\left[\begin{array}{llllll}
90.0000 & 124.3590 & 83.1176 & 33.2787 & 8.1583
\end{array}\right]
\end{aligned}
$$




\section{Experimental Research}

An experimental prototype of flow-coupled secondary regulation system was established (see in Figure 2) and experimental researches had been conducted in order to validate the functionality of the controller. The load cylinder velocity with LQR controller in case of different input is shown in Figure.3. Meanwhile, curve 13 and 4 is the load cylinder velocity response when the input is $1-2,4$ and 8 voltage.Figure. 4 plots the load velocity comparisons between using LQR controller and PID controller. Meanwhile, curve 1 and 3 is the load velocity using PID controller when the input is 8 and 1 voltage. Curve 2 and 4 is the load velocity using LQR controller when the input is 8 and 1 voltage. From the Figure.3, we can obtain that the load cylinder velocity is proportional to the input and the response time is less than 2 second after using LQR controller. Meanwhile, the system can be controlled exactly. The Figure. 4 shows that the LQR controller and PID controller are effective when the input is 1 voltage and the response time with PID controller is reduced to 1.5 second. Meanwhile, we can obtain that the LQR controller is better than PID controller when the input is 8 voltage From Figure.4. The response time with PID controller achieves to 3 second and the overshoot is more than $15 \%$. However, the overshoot is nearly free with LQR controller.

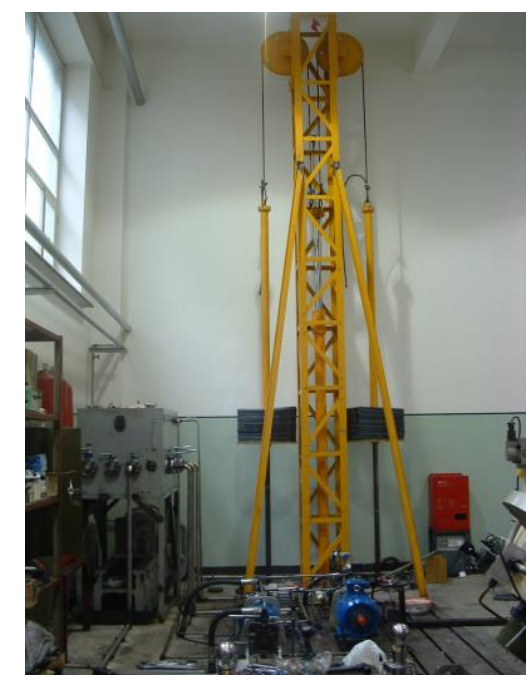

Figure. 2 Experimental Prototype of Flow-Coupled Secondary Regulation System

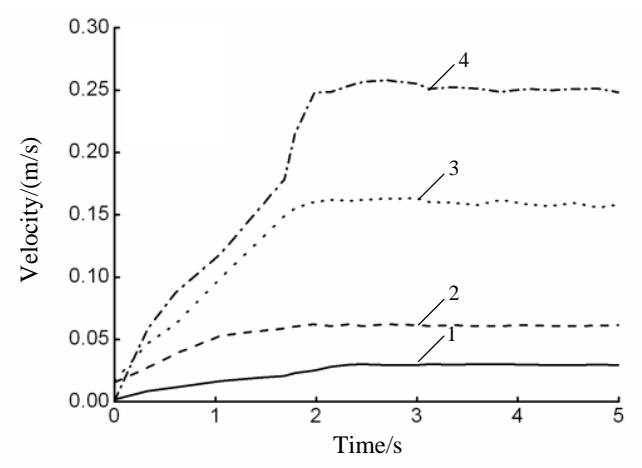

Figure 3. Load Cylinder Velocity with LQR Controller in Case of Different Input 


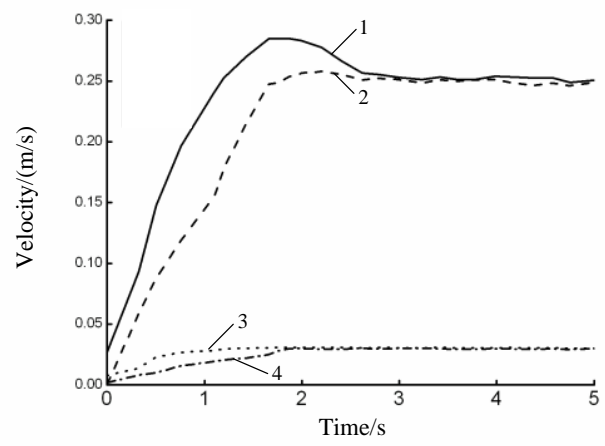
Figure 4. Load Cylinder Velocity Comparisons between Using LQR
Controller and PID Controller

\section{Conclusion}

From the experimental results, we can know that the exact linearization controller is very effective and suitable to the system, which fully verifies the system is nonlinear in nature. Furthermore, The LQR controller based on the nonlinear description-variable model is irrelevant to the input. The PID controller based on the linearized model is effective near the zero point. But, the LQR controller is better than the PID controller when the system works far from the zero point. With the development of the nonlinear control theory, the nonlinear system can be synthesized by means of the nonlinear control theory, but the increment linearization.

\section{Acknowledgements}

This work was supported in part by National Natural Science Foundation of China under Grant No. 61201266.

\section{References}

[1] X. Yang, "Energy Recovery Efficiency of Flow-coupled Flywheel Vehicle," Research Journal of Applied Sciences, Engineering and Technology, vol. 4 no. 17, (2012), pp. 2905-2910.

[2] S. Liu, Y. Yao and T. Shang, "Full Hydraulic Drive System of Minitype Loader Based on Secondary Regulation Technique," Journal of Jilin University (Engineering and Technology Edition), vol. 41 no. 3, (2011), pp. 665-669.

[3] J. Guo, L. Zhang and X. Cai, "Exact Linearization Control Scheme of DFIG," Electric Machines and Control, vol. 13 no. 1, (2009), pp. 57-62.

[4] H. T. Le, S. R. Lee and C. Y. Lee, "Integration Model Reference Adaptive Control and Exact Linearization with Disturbance Rejection for Control of Robot Manipulators," International Journal of Innovative Computing, Information and Control, vol. 7 no. 6, (2011), pp. 3255-3267.

[5] Na W. K. and Diong B., "Nonlinear Control of PEM Fuel Cells by Exact Linearization," IEEE Transactions on Industry Applications, vol.43 no. 6, (2007), pp. 1426-1433.

[6] Z. Gao, X. Zhang and H. Lin, "Exact Feedback Linearization Control to BUCK Converter with Constant Power Loads," Control and Decision, vol. 24 no. 7, (2009), pp. 1117-1120.

[7] J. Wu and J. Zhang, "Improved Control Based on Two-Wheeled Self-balancing Robot LQR Controller," Journal of Harbin University of Science and Technology, vol. 17 no. 6, (2012), pp. 1-5. 
International Journal of Multimedia and Ubiquitous Engineering

Vol.10, No.6 (2015) 